\title{
Siniestralidad laboral en clave de género
}

\section{Labor accidents according to gender}

\section{Ignacio Fontaneda González ${ }^{1}$}

'Universidad de Burgos, Burgos, España.

Fechas - Dates

Recibido: 2020.04.25

Publicado: 2020.07.15
Sección coordinada por / Section Coordinator

Dr. Guillermo García González

Profesor Titular Derecho del Trabajo y SS Universidad Internacional de La Rioja (UNIR)

Email: guillermo.garcia@unir.net 


\section{Entrevista con Ignacio Fontaneda González}

1. ¿Cuál es la procedencia, especialización y áreas de trabajo de los autores del artículo? ${ }^{(1)}$ ¿Qué características le parecen más destacables de este grupo de trabajo?

Los autores pertenecen al Grupo de Investigación de la Universidad de Burgos iGR (Ingeniería y Gestión Responsable) en colaboración con el profesor Dale O’Ritzel de la Southern Illinois University.

Este grupo de trabajo viene realizando aportaciones en el campo de la seguridad laboral durante los últimos 20 años, especialmente en el campo del estudio y prevención de accidentes laborales.

Algo destacable en el grupo es la presencia de gran diversidad de perfiles, con estudios en Ingeniería, Derecho, Economía y Psicología. Lo que hace de él un grupo multidisciplinar, con potencial para observar los problemas desde distintos enfoques.

\section{2. ¿Cómo se ha financiado el estudio?}

Este estudio no ha recibido financiación. Hemos recibido los microdatos de accidentes, de la Subdirección General de Estadísticas del Ministerio de Trabajo y Seguridad Social, para la realización de los análisis.

\section{3. ¿Qué problema pretende abordar este estudio y dónde radica su interés o relevancia para la seguridad y salud en el trabajo?}

Este estudio pretende profundizar en los factores que influencian la duración de las bajas según determinados factores recogidos en el parte de accidente de trabajo, como son: el sexo, edad de la persona accidentada, el tipo y lugar de trabajo, el tamaño de la empresa, el día de la semana, la hora del accidente y las horas que el trabajador lleva trabajando.

La duración de las bajas, o los días sin trabajar, además de reflejar la gravedad del accidente, influencian los costes, tanto para el trabador, para quien indemniza la baja y para la empresa, por el coste, muchas veces no recogido y suficientemente ponderado de la sustitución del trabajador que está de baja.

La relevancia del estudio viene determinada por el hecho de que se analizan un total de 4.188.714 accidentes (un gran número), ocurridos entre 2008 y 2014.

\section{4. ¿Qué aporta este estudio de novedoso o destacable en relación al resto de producción científica sobre el problema estudiado?}

El estudio es robusto, por la gran cantidad de accidentes incluidos en su análisis, lo que hace que se puedan identificar diferencias significativas.

El estudio deja patente como las mujeres presentan bajas con mayor duración media que los hombres y que la duración de las bajas se incrementa con la edad, dato muy relevante, teniendo en cuenta el envejecimiento que se está produciendo en la población trabajadora. 
También las bajas tienden a tener más duración en las empresas de menos de cinco trabajadores.

Y algo destacable es como la duración media de las bajas va aumentando conforme avanza la semana, siendo la duración más baja los lunes y subiendo progresivamente, para ambos sexos, hasta el viernes.

Como cabía esperar las bajas son más altas en los desplazamientos que en el lugar habitual de trabajo. Estos incluyen accidentes de tráfico que tienden a ser más graves.

\section{5. ¿Se han encontrado con alguna dificultad o contratiempo para el desarrollo del estudio?}

Ningún contratiempo relevante.

\section{6. ¿Se han obtenido los resultados esperados o se ha producido alguna sorpre- sa?}

Es conocido el efecto lunes, una mayor notificación de accidentes los lunes. Pero es novedoso el conocer como los accidentes de los lunes tienden a ser menos graves (con menor duración de las bajas) y esta va subiendo a lo largo de la semana; menos accidentes, pero más graves conforme avanza la semana.

También llama la atención como la duración de las bajas, en accidentes que se producen fuera del lugar habitual de trabajo, son más altas que en el trabajo habitual.

\section{7. ¿Cómo ha sido el proceso de publicación? ¿Han sufrido alguna incidencia?}

Ninguna incidencia relevante.

\section{8. ¿Qué implicaciones tiene este estudio para la prevención de riesgos labora- les? ¿Cuál sería la recomendación para mejorar la práctica profesional en rela- ción al problema estudiado?}

El conocimiento de los factores que afectan a la duración de las bajas debidas al accidente, variable íntimamente relacionada con los costes del accidente, puede ayudar a reducir estas duraciones y los costes asociados.

Las aseguradoras y la Seguridad Social pueden encontrar en un mejor conocimiento de los factores que afectan a las bajas una mejor previsión de los costes asociados según el tamaño de las empresas y las características de sus trabajadores.

El hallazgo de la fuerte relación de la duración de las bajas con la edad del accidentado, especialmente en el caso de las mujeres mayores de 55 años, donde la duración media de las bajas aumenta en mayor medida, debe ayudar a poner el foco en la recuperación de estos trabajadores y en una mejor preparación para hacer frente a las consecuencias del accidente. Con la edad puede ser recomendable establecer rutinas de ejercicio que permitan a la población trabajadora estar en mejor forma, lo que redundará en una mayor calidad de vida, además de una mejor recuperación después de un accidente. 
La mayor gravedad del accidente conforme avanza la semana puede tener relación con la fatiga, que se va acumulando a lo largo de la semana. Ahora que se está planteando recortar las horas trabajadas a la semana, puede ser aconsejable reducir la duración de la jornada en mayor medida conforme avance la semana.

También parece interesante incrementar la labor preventiva cuando un trabajador va a realizar una tarea fuera de su lugar de trabajo habitual o un trabajo que no es el habitual. En estos casos, además de aumentar la probabilidad de accidente también se incrementa su gravedad, medida en jornadas perdidas.

\section{Resumen del artículo(1)}

En este trabajo se analizan los factores que influyen en la duración de las bajas tras un accidente laboral. Son analizados 4.188.714 accidentes ocurridos en España entre 2008 y 2014.

El gran número de accidentes analizados permite encontrar diferencias estadísticamente significativas, por lo que las conclusiones no se deben al azar. Siendo esta significación estadística una aportación relevante frente a otros estudios donde se podía intuir esta relación, pero no podía demostrarse de modo estadísticamente significativo, por el reducido tamaño de las muestras estudiadas.

El objetivo del estudio era establecer la posible relación entre la duración de las bajas debidas a accidente laboral y distintas variables recogidas en el parte oficial de accidente de trabajo, como son la edad, el género, el tamaño de la empresa, el día de la semana, el tipo de trabajo (habitual o no) y el lugar de trabajo. De esta forma, las principales conclusiones son:

- La duración de las bajas se incrementa de forma sostenida, en su valor medio, conforme se incrementa la edad del trabajador accidentado.

- Las jornadas perdidas cuando la persona accidentada es una mujer, de media, son superiores a las perdidas cuando el accidentado es un hombre. Esta afirmación se sostiene para distintas edades, días de la semana (entre semana), realizando el trabajo habitual y en empresas de distintos tamaños.

- La duración de las bajas se incrementa conforme avanza la semana, siendo la duración de las bajas de los accidentes producidos en lunes menor que la de los martes, esta menor que la de los miércoles y así sucesivamente jueves y viernes.

- Las jornadas perdidas por el accidente son de media mayores para los accidentes producidos cuando se realiza un trabajo distinto del habitual.

- La duración de las bajas, según el lugar donde se produce el accidente, es mayor en los accidentes in itinere, seguido de los accidentes en misión, en otro centro distinto del habitual. Siendo la duración menor en los accidentes producidos en el centro de trabajo. 
Teniendo en cuenta los resultados alcanzados se recomienda:

- Establecer protocolos y actuaciones para mantener en mejor forma a los trabajadores más mayores, como colectivo más vulnerable, además de trabajar en tratamientos más efectivos para una mejor recuperación de estos trabajadores. También se puede plantear recortar la jornada laboral conforme se incrementa la edad, pues la fatiga parece ser un factor en la gravedad del accidente que afecta en mayor medida a los más mayores.

- Además, se deben establecer pautas diferenciales en la prevención de accidentes y en la evaluación de riesgos en función de las características del trabajador, el trabajo y la empresa.

- Especial atención merecen los desplazamientos, además de los trabajos en lugares distintos del habitual, por la mayor gravedad de los accidentes asociados, medidos en días de baja.

\section{Referencias}

1. Fontaneda I, Camino López MA, González Alcantara OJ, Ritzel DO. Gender differences in lost work days due to occupational accidents. Safety Sci. 2019;114:2329. doi:10.1016/j.ssci.2018.12.027 